\title{
Adaptação editorial para leitores com dislexia
}

\author{
Editorial adaptation for dyslexic readers
}

Thalita Albino Machado, Juliana Bueno

dislexia, dificuldade de leitura, design editorial, design inclusivo

Publicações brasileiras geralmente apresentam diversos empecilhos para pessoas diagnosticadas com dislexia, cuja leitura é marcada por distorções, substituições ou omissões, resultando em erros na compreensão. Essa condição costuma afastar jovens disléxicos da leitura recreativa, conhecida por sua influência positiva no hábito de leitura e até no gosto pelo estudo. A partir dessa realidade, este Trabalho de Conclusão de Curso em Design Gráfico, na Universidade Federal do Paraná, pautou-se na metodologia Design Thinking (Ambrose \& Harris, 2010), a qual foi utilizada para: (1) estudar as áreas de Design da Informação, Editorial e Inclusivo, Dislexia e Design para Dislexia; (2) realizar uma pesquisa de campo para compreender as dificuldades e hábitos de leitura dos usuários; (3) reunir diretrizes de produção de editoriais acessíveis para o público disléxico; (4) validar a aplicação das recomendações com o público-alvo. Assim, com o percurso metodológico foram gerados 18 requisitos e 13 restrições de Design Editorial para a produção de materiais acessíveis para esse público. Os quais foram aplicados na adaptação editorial de parte do livro "Percy Jackson e o Ladrão de Raios", de Rick Riordan, cujo enredo da narrativa é considerado uma metáfora que retrata jovens disléxicos de forma positiva.

dyslexia, reading disoder, editorial design, inclusive design

Brazilian publications usually present several obstacles for people diagnosed with Dyslexia, whose reading is marked by distortions, substitutions or omissions, resulting in comprehension mistakes. This condition tends to keep young dyslexic people away from reading for fun, known for its positive influence on the reading habit and even on the affinity for studying. From on this reality, on a final dissertation submitted for the Degree in Graphic Design, on the Federal University of Paraná, 18 requirements and 13 restrictions of Editorial Design for the production of accessible materials for the dyslexic audience were generated. Design Thinking methodology (Ambrose \& Harris, 2010) was used to (1) study the areas of Informational, Editorial and Inclusive Design, Dyslexia and Design for Dyslexia; (2) conduct a field research to comprehend the users' difficulties and reading habits; (3) generate guidelines of editorial productions for the dyslexic audience; (4) validate the application of the recommendations with the targeted audience. The final product was part of Rick Riordan's book "Percy Jackson and the Lightning Thief", whose plot is considered a metaphor that portrays young people with dyslexia positively.

\section{Introdução}

A dislexia é uma condição neurobiológica que resulta em dificuldade para reconhecer, soletrar e codificar textos (IDA, 2002). Esse Transtorno Específico de Aprendizagem atinge, de forma severa, cerca de $10 \%$ das crianças em idade escolar, e até $25 \%$ em níveis mais leves, segundo Capovilla (2008). Além de interferir significativamente na performance escolar das crianças

Anais do $10^{\circ} \mathrm{CIDI}$ e $10^{\circ} \mathrm{CONGIC}$

Kelli C.A.S. Smythe, Rafael de Castro Andrade (orgs.)

Sociedade Brasileira de Design da Informação - SBDI

Curitiba | Brasil | 2021
Proceedings of the $10^{\text {th }} \mathrm{CIDI}$ and $10^{\text {th }}$ CONGIC

Kelli C.A.S. Smythe, Rafael de Castro Andrade (orgs.)

Sociedade Brasileira de Design da Informação - SBDI Curitiba | Brazil | 2021 
diagnosticadas, costuma afastá-las da leitura de livros por lazer, o que pode prejudicar o desenvolvimento do hábito de leitura e até o gosto pelo estudo (Cardoso, 2018).

Para Sykes (2008), os conhecimentos de Design Editorial fornecem uma perspectiva única sobre como criar textos acessíveis para esse público. Gruszynski (2015) afirma que devemos considerar o Design como uma atividade que lida especialmente com a informação. Nesse contexto, as áreas de Design Editorial, Design da Informação e Design Inclusivo se tornam agentes da leitura.

A partir disso, evidencia-se a necessidade de tornar a edição de livros mais inclusiva para leitores com dislexia. A leitura recreativa poderia se tornar um estímulo para melhores hábitos de leitura ao ser apresentada em um material que leva em consideração as especificidades de decodificação de textos do público disléxico.

Na revisão de literatura, foi observado que as soluções de pesquisas brasileiras a respeito de design e dislexia apresentam apenas resultados dependentes de dispositivos digitais. Apesar de eficiente, esse recurso não é viável a todos os contextos pois o acesso a um e-book, por exemplo, depende de um letramento digital, como aponta Gruszynski (2015).

Assim, objetivou-se conceituar recomendações e contraindicações de Design Editorial na língua portuguesa brasileira com foco no público disléxico e no escopo de materiais impressos.

Este trabalho buscou favorecer a inclusão social dos indivíduos com dislexia, desenvolvendo uma adaptação editorial para leitores portadores de dislexia, através da diagramação fundamentada em diretrizes de adaptação e conceitos de Design Gráfico.

\section{Metodologia}

Neste artigo, optou-se por apresentar o referencial teórico intrínseco ao percurso metodológico do TCC. A metodologia escolhida para definição e aplicação dos requisitos foi o Design Thinking, de Ambrose e Harris (2010), com fases bem delimitadas, mas com a liberdade necessária de adaptação e escolha de ferramentas dentro de cada uma.

\section{Etapa 1: Definir}

Definição e compreensão das problemáticas e demarcação do público-alvo. Para isso, foram consultados os trabalhos de Capovilla (2008), Assunção (2018), Maceri (2003) e da Associação Internacional de Dislexia (2002).

\section{Etapa 2: Pesquisar}

Os princípios de Design Inclusivo (Connell et al., 1997), como uso equitativo, flexibilidade no uso e informação perceptível, complementaram as recomendações de Design Editorial estudadas. Nas contribuições existentes do Design Gráfico para o problema foram identificadas as diretrizes de Design para Dislexia utilizadas, sendo as principais fornecidas pelo Dyslexia Style Guide (BDA, 2018), por Maceri (2003) e Pedley (2006 apud Campêlo, 2013). 
As recomendações de Schriver (1997) consideram grande parte das diretrizes encontradas. Entretanto, alguns itens contrapõem algumas das ideias convencionadas. Se, para o público não disléxico, estilizar palavras em itálico cumpre o papel de ênfase, para os leitores com dislexia isso resulta em aglomeração visual das palavras. O efeito visual de continuidade das fontes serifadas resulta na mesma impressão de agrupamento.

Em seguida, foi realizada a pesquisa de campo com fonoaudióloga e público-alvo (questionários online) e a análise de similares. O questionário com a especialista abordou questões sobre o tratamento da dislexia, materiais adaptados e relevância da literatura infantojuvenil como estímulo à leitura. Perguntas sobre o efeito da dislexia na vida acadêmica, seus hábitos de leitura e preferência entre livros impressos e digitais, entre outras, estavam no questionário para o público. Para análise dos similares, foi utilizado o modelo de análise gráfica de Mijksenaar (1997), adaptado por Heleno e Reque (2017) ${ }^{1}$ e pela autora.

\section{Etapa 3: Idealizar}

As recomendações de Design Editorial e Inclusivo, as diretrizes de acessibilidade para Dislexia, as conclusões da pesquisa de campo e da análise de similares resultaram em tabelas de requisitos e restrições de adaptação. As diretrizes foram divididas nas quatro categorias da análise de similares.

\section{Etapa 4: Prototipar}

Para validar o material com o público-alvo, os requisitos foram empregados no redesign do livro de ficção "Percy Jackson e o Ladrão de Raios", de Rick Riordan. Segundo Wielewicki e Tadano Filho (2012), as aventuras narradas na saga são uma grande metáfora sobre as dificuldades vividas pelos indivíduos diagnosticados com dislexia e outros transtornos de aprendizagem.

\section{Etapa 5: Selecionar}

Nessa etapa, foi possível validar o material adaptado. Com base nas sugestões dos entrevistados, foi adicionado à capa um guia de leitura inspirado no tiposcópio, um auxílio para usuários com baixa visão que facilita a localização do leitor (Acessibilidade na Prática, 2015).

\section{Etapa 6: Implementar}

Após refinamento do material e das diretrizes, os 5 primeiros capítulos da obra selecionada foram adaptados, impressos e disponibilizados em plataforma online ${ }^{2}$. O objetivo da estrutura editorial do produto final é a possibilidade de aplicação em qualquer material editorial adaptado para o público com dislexia.

\footnotetext{
${ }^{1}$ Modelo de análise dividido em 4 categorias: descrição, diferenciação, hierarquia e apoio. Fonte: Heleno e Reque (2017).

${ }^{2}$ Disponível em: <https://issuu.com/thalita.machado/docs/percy_jackson_e_o_ladr_o_de_raios_-_adaptado_para_> Acesso em: 27 abr, 2021
} 


\section{Etapa 7: Aprender}

Através da análise de resultados do projeto, foi observado se a solução apresentada alcançou os objetivos definidos e as possibilidades de continuidade.

\section{Resultados}

Com base nas recomendações da pesquisa teórica, em conjunto com as sugestões dos entrevistados e as conclusões da análise de similares, foram estabelecidos 18 requisitos (Tabela 1) e 13 restrições (Tabela 2) para que o propósito definido fosse cumprido.

A síntese desses dados está apresentada nas tabelas a seguir, separadas nas categorias do modelo de análise.

Tabela 1: Requisitos. Fonte: as autoras.

\begin{tabular}{llll}
\hline Descrição & $\begin{array}{l}\text { Fundamentação } \\
\text { teórica }\end{array}$ & $\begin{array}{l}\text { Pesquisa de } \\
\text { campo }\end{array}$ & $\begin{array}{l}\text { Análise de } \\
\text { similares }\end{array}$ \\
\hline $\begin{array}{l}\text { Dimensões de maior aproveitamento } \\
\text { do papel; }\end{array}$ & & $\checkmark$ \\
Formato impresso e digital; & & $\checkmark$ \\
Papel fosco; & $\checkmark$ & \\
Cor do papel: off-white ou creme; & $\checkmark$ & \\
\hline
\end{tabular}

\section{Diferenciação}

Tipografia sem serifa e com altura-x elevada: Arial, Verdana, Tahoma, Calibri, Open Sans ou Helvetica;

Tamanho de fonte entre 12 e 14 pontos;

Tracking de aproximadamente $35 \%$ da largura das letras;

Espaçamento extra entre parágrafos e títulos;

Alto contraste entre texto e fundo;

\section{Hierarquia}

Títulos com fonte diferenciada e/ou pelo menos $20 \%$ maiores do que o texto;

Espaçamento entre palavras $=3,5$ vezes o espaçamento entre caracteres; 
Entrelinhas proporcionais ao espaço

entre palavras, pelo menos $25 \%$ do

tamanho das letras;

Até 60 caracteres por linha;

Alinhamento à esquerda;

Margens de tamanho mínimo $2 \mathrm{~cm}$;

Ênfase com negrito;

$\checkmark$

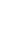


O produto final deste TCC foi o editorial adaptado dos primeiros capítulos do livro "Percy Jackson e o Ladrão de Raios". A fonte Open Sans foi utilizada em todo o projeto. Na figura 1, as formatações tipográficas e as modificações no uso do itálico são apresentadas.

A adaptação da parte externa do livro envolveu mudanças na lombada, quarta capa e orelhas do livro. Como ilustrado na figura 2, o livro adaptado foi impresso e encadernado.

Figura 1: Tipografia do miolo do livro adaptado. Fonte: as autoras.

Tipografia da versão adaptada

\begin{tabular}{|c|c|c|c|}
\hline \$ Capítulo 1 & Open Sans Bold & Olhe, eu não queria ser um meio-sangue. & \\
\hline $\begin{array}{l}\text { Sem querer, transformo } \\
\text { em pó minha professora } \\
\text { de iniciação à álgebra }\end{array}$ & $\begin{array}{l}15,6 \mathrm{pt} \\
\text { Entrelinha: } 20 \% \\
\text { Tracking: } 50\end{array}$ & $\begin{array}{l}\text { Se você está lendo isto porque acha que } \\
\text { pode ser um, meu conselho é o seguinte: } \\
\text { feche este livro agora mesmo. Acredite } \\
\text { em qualquer mentira que sua mãe ou } \\
\text { seu pai lhe contou sobre seu nascimento, } \\
\text { e tente levar uma vida normal. }\end{array}$ & $\begin{array}{l}\text { Open Sans Regular } \\
13 \mathrm{pt} \\
\text { Entrelinha: } 30 \% \\
\text { Tracking: } 25\end{array}$ \\
\hline
\end{tabular}

Itálicos (ênfase, idiomas estrangeiros e onomatopeias)

Original

—O Zen kai alloi theoi! — gritou ele. — Está bem arrás de mim! Você não conton a ela?

A làmina de metal atingiu o ombro dela e passou direto por seu corpo, como se ela fosse feita de água: Zaz!
Adaptado

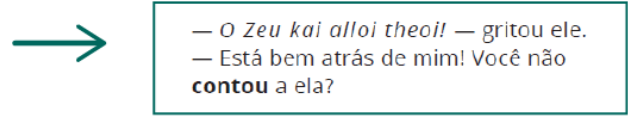

A lâmina de metal atingiu o ombro dela e passou direto por seu corpo, como se ela fosse feita de água: "Zaz!"

Legenda de textos com fontes de contextualização na versão adaptada

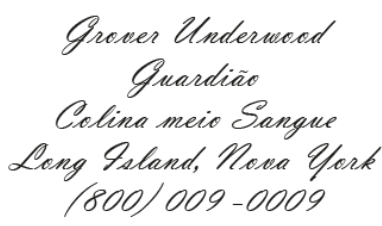

(Grover Underwood / Guardião, Colina Meio-Sangue/ Long Island, Nova York / (800) 009-0009)
Trecho em fonte diferenciada para contextualização e
dinamismo da narrativa 
Figura 2: Livro adaptado em contexto de uso com guia de leitura. Fonte: as autoras.

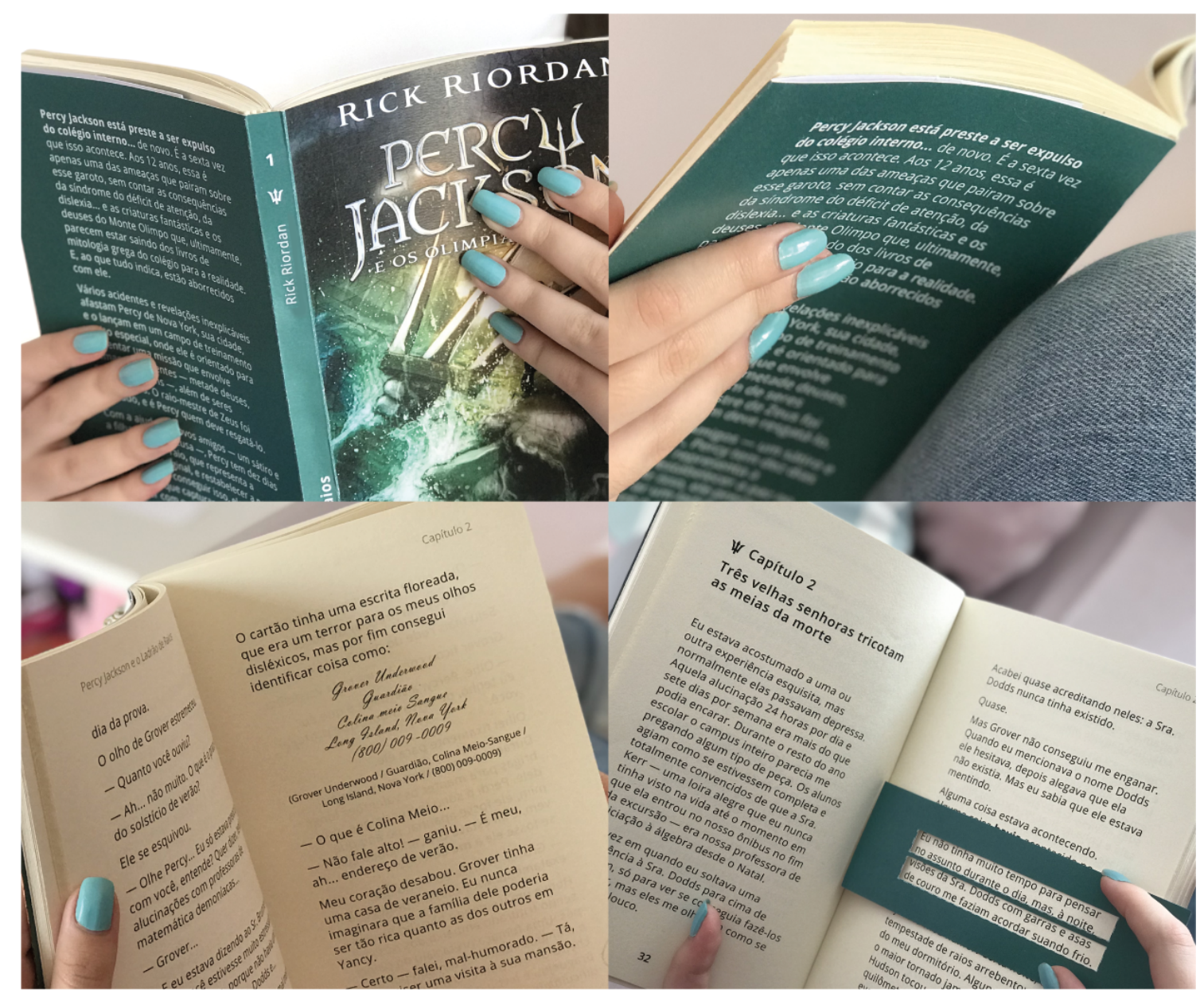

\section{Considerações Finais}

Com esta pesquisa, procurou-se oferecer, além de uma leitura com menos obstáculos ao público com dislexia, um modelo de requisitos editoriais para projetos de design inclusivos para esses leitores.

Este trabalho possibilitou a compreensão dos diversos obstáculos causados por esse transtorno de aprendizagem. A pesquisa teórica esclareceu em que contexto projetos de design gráfico e editorial poderiam colaborar. A consulta aos leitores disléxicos propiciou o entendimento de que, ainda que uma solução editorial não resolva todos os problemas causados pela dislexia, estratégias de leitura pessoais dos usuários são facilitadas quando o produto é projetado de forma inclusiva.

$\mathrm{Na}$ área de atuação do Design Gráfico Inclusivo ainda há necessidade de projetos de inclusão voltados para o público com dislexia. Por isso, almeja-se que os requisitos e restrições conceituados possam ser aplicados em diferentes contextos. Acredita-se que as soluções defendidas são relevantes para o desenvolvimento de outros produtos gráficos, como livros didáticos, revistas, e-books e sites. 


\section{Referências}

Ambrose, G., Harris, P. (2010). Basics Design 08: Design Thinking. Lausanne: AVA Publishing SA.

Assunção, G. S. (2018). A Dislexia e os Desafios no Processo de Aprendizagem. Monografia (Graduação), Universidade do Estado da Bahia, Santo Antônio de Jesus.

British Dyslexia Association (BDA). (2018). Dyslexia Style Guide 2018: Creating Dyslexia Friendly Content.

Campêlo, R. A. (2013). Acessibilidade para portadores de dislexia em um ambiente virtual de aprendizagem móvel. Dissertação (Mestrado), Universidade Federal de Pernambuco, Recife.

Capovilla, A. G. S. (2008). Dislexia no desenvolvimento: definição, avaliação e intervenção. In: Transtorno de aprendizagem da avaliação à reabilitação. São Paulo: Editora Artes.

Cardoso, R. (2018). Importância da literatura infanto-juvenil no contexto escolar. Pedagogia In Revista, pp. 11-19.

Connell, B. R. et al. (1997) The Center for Universal Design - Universal Design Principles. The Principles of Universal Design, Raleigh, NC, The Center for Universal Design. Disponivel em: https://projects.ncsu.edu/ncsu/design/cud/about_ud/udprinciplestext.htm

Gruszynski, A. C. (2015). Design editorial e publicação multiplataforma. Intexto, v. 0, n. 34 , p. 571.

Heleno, L. L.; Reque, M. (2017) Livro Infantil Adaptado à Criança Surda: Estimulando o Letramento. TCC (Graduação), Universidade Federal do Paraná, Curitiba.

International Dyslexia Association (IDA). (2002). Definition of Dyslexia. Disponível em https://dyslexiaida.org/definition-of-dyslexia/

Maceri, K. (2003). Document Design for Users with Reading Disorders. Artigo, The University of Memphis, Memphis.

Mijksenaar, P. (1997) Visual Function: An Introduction to Information Design. Rotterdam, 010 Publishers.

Schriver, K. A. (1997). Dynamics in Document Design: Creating Text for Readers. New York: Wiley.

Sykes, J. (2008). Dyslexia, Design and Reading: Making Print Work for College Students with Dyslexia A Qualitative Interaction Design Study. Tese (Doutorado), Carnegie Mellon University, Pittsburgh.

Wielewicki, V. H. G.; Tadano Filho, M. (2012). TDAH e Dislexia em Percy Jackson. Darandina, v. 5 , n. 2, pp. 1-17.

\section{Sobre as autoras}

Thalita Machado, UFPR, Brasil <thalimachado.tm@gmail.com> Juliana Bueno, Dra., UFPR, Brasil <julianabueno.ufpr@gmail.com> 